\title{
Roads to the strategic targeting of ovarian cancer treatment
}

\author{
Griselda Irusta \\ Laboratorio de Fisiología y Biología tumoral del Ovario, Instituto de Biología y Medicina Experimental (IByME- \\ CONICET), Buenos Aires, Argentina
}

Correspondence should be addressed to G Irusta; Email: irustag@gmail.com

\begin{abstract}
Although ovarian cancer mortality rates have slightly declined in the last $\mathbf{4 0}$ years, ovarian cancer continues to be the eighth cause of cancer death in women. Ovarian cancer is characterized by its high response to treatments but also by its high rate of recurrence. Although treatments are limited to cytoreductive surgery and platinum-based chemotherapy, other therapies using antiangiogenic agents and poly (ADP-ribose) polymerase inhibitors are being tested. Nevertheless, these therapeutic strategies have had poor results and new potential targets and approaches are thus needed. The present review focuses on the recent evidence on antiangiogenic strategies in ovarian cancer cells and on the mechanisms governed by Notch and $\beta$-catenin proteins. It also describes the concept of 'vascular normalization' by using the platelet-derived growth factor, PDGFB, molecule as a tool to regulate ovarian tumor angiogenesis and thus improve ovarian tumor treatment. It has been reported that alterations in the Notch system components and changes in the canonical Wnt/ $\beta$-catenin signaling, the other pathway of our interest, are relevant to molecular events that contribute to ovarian cancer development. Thus, in this review, we consider these aspects of the ovarian tumor biology as potential new therapeutic strategies for the treatment of this disease.

Reproduction (2021) 161 R1-R11
\end{abstract}

\section{Introduction}

\section{Why is ovarian cancer complicated?}

William Shakespeare wrote in his novel Hamlet: There are more things in Heaven and Earth, Horatio, than are dreamt of in your philosophy, suggesting that human knowledge is limited. Nevermore correct than when we talk about ovarian cancer. Ovarian cancer comprises different tumor types grouped under a single denomination. Although these histological subtypes share some features, they are different enough to have different clinical features as well as treatments and genetic characteristics. These differences lie in their etiology, which is sometimes outside the ovary and often unknown. The different origins of ovarian cancer give rise to tumors in different contexts. This makes this 'apparently single entity' to behave as multiple ones.

When we refer to ovarian cancer statistics, we have to take into account the specific types of ovarian cancer included for the statistics and the level of cancer registration. The 5-year survival rate reported worldwide is highly variable as both the risk and mortality rates are affected by many variants, including the ethnicity, tumor types, and age profiles. In addition, the incidence and mortality due to ovarian cancer are hard to estimate because only few countries have high-quality data. However, what is common to all continents is that the mortality due to ovarian cancer is close to $50 \%$ of the incidence (GLOBOCAN study). The GLOBOCAN study has predicted that, by 2035, there will be a worldwide increase of $55 \%$ in the incidence of ovarian cancer and an increase in ovarian cancer deaths of $67 \%$ based on population increase.

Ovarian cancer is the seventh most common cancer and the eighth most common cause of death from cancer in women in the world. Although the most common 'women's cancer' is breast cancer, the mortality due to ovarian tumors is higher. This raises the need to gain deeper knowledge about the mechanisms that govern the development of ovarian cancer. One of the intriguing characteristics of ovarian cancer is the unknown etiology, which, together with confusing characteristics of these tumors, leads to the late detection of the illness. The high-grade serous carcinoma, for example, is now strongly suggested to be originated from the fimbriated end of the fallopian tube. These tumors are classified as type II carcinomas and their late detection agrees with the peritoneal seeding by malignant cells from the fimbriated end of the tube. In contrast, the low-grade serous carcinoma seems to arise from borderline serous carcinoma or endometriosis (Przybycin et al. 2010, Wang et al. 2013). These tumors, which are detected early and are low-grade tumors, are classified as type I carcinomas (Przybycin et al. 2010), a category that also includes mucinous, endometroid and clear cell carcinomas (Torre et al. 2018). This indicates that the 
site of origin of the different histotypes of ovarian cancer is related to their malignancy and stage of detection. The different ovarian cancer origins and molecular characteristics mostly contribute to the high complexity of this illness.

\section{Ovarian cancer treatment}

A search in the Pubmed database using the terms 'ovarian cancer and treatment' in the past 10 years displayed close to 27,400 research papers vs the same search with the terms 'breast cancer and treatment', which displayed 108,000 research papers. The smaller number of publications related to ovarian cancer treatment compared to breast cancer treatment, together with the poor improvement in the cure of these tumors, evidences the need for new approaches for this cancer type.

Beyond all the research being performed in ovarian cancer therapeutics, surgery is still a mainstay in staging and treatment of ovarian cancer. When the tumors are in advanced stages, which is the most usual situation, the surgical approach to determine the real extent of the disease and the stage according to the International Federation of Gynecology and Obstetrics (FIGO) classification and to establish the best surgical techniques is an open laparotomy (Santaballa et al. 2016). Unequivocally, cytoreductive surgery is associated with increased survival and the volume of residual disease remaining after the surgery is inversely correlated with survival (Santaballa et al. 2016).

The first recommended treatment after surgery depends on the FIGO stage at the time of detection and the histotype of the tumor. Besides these considerations, for early stages, the traditional therapy consists of platinum-based chemotherapy with carboplatin and paclitaxel, whereas, for advanced stages, therapy consists of variations of this regimen and intraperitoneal chemotherapy. The Gynecologic Oncology Group (GOG) has conducted three large randomized, phase III clinical trials of intraperitoneal chemotherapy, known as GOG 104, 114, and 172, which have shown that intraperitoneal chemotherapy is significantly superior to intravenous one. Apart from the platinum-based therapy, patients who are not recommended to receive paclitaxel receive pegylated liposomal doxorubicin (PLD) (Pignata et al. 2011). Also, some patients are treated with an antiangiogenic therapy with bevacizumab, a humanized $M A B$ directed against vascular endothelial growth factor (VEGF).

In 1960, Folkmanand Beckerelucidated theimportance of vascularization for tumor growth. These authors implanted microscopic-sized tumors in the perfused thyroid gland of mice and observed that these tumors grew more than 1000 times than the original tumors in isolated organs and were highly neovascularized. This suggested that, in the absence of neovascularization, tumors would stop growing (Folkman et al. 1963). Later, in 1971, Folkman demonstrated the hypothesis that 'tumor growth is angiogenesis dependent' (Folkman 1971). In that publication, this author showed that, in the rabbit eye, tumor dormancy at a microscopic size was due to the blocked angiogenesis of tumors. After this publication, Folkman's hypothesis has been reinforced by many published data. This has opened a new field with new bioassays regarding angiogenesis and, consequently, new molecules, such as monoclonal antibodies, endogenous peptides, small organic molecules and miRNA, being considered.

Bevacizumab (Avastin) was approved by the Food and Drug Administration (FDA) for colorectal cancer treatment on February 26, 2004, and is the first antiangiogenic drug to receive FDA approval. As aforementioned, this drug inhibits VEGF, which is the main protein involved in inducing angiogenesis (Leung et al. 1989). With the 1900s idea of inhibiting the formation of new blood vessels to block tumor growth, VEGF turned to be an attractive target for this therapeutic strategy. In this line, the observations from the clinical trial that showed the anticancer effect of bevacizumab in colorectal cancer became very interesting. The comparison in the median survival time between patients treated with the irino tecan/5-fluorouracil/leucovorin (IFL) chemotherapy and bevacizumab and the patients treated with IFL and placebo showed that those receiving bevacizumab had a median end-point survival time of 5 months longer than those without. This difference between treatments was the highest ever found in the median survival time in a phase III clinical trial. This observation is in accordance with the hypothesis that bevacizumab works as a promoter of vascular stabilization as described subsequently (Goodman 2004). Based on this evidence, on June 13, 2018, bevacizumab was approved by the FDA as first-line treatment in epithelial ovarian, fallopian tube, and primary peritoneal cancer stage III or IV in combination with carboplatin and paclitaxel (Marchetti et al. 2019). In their study, Marchetti et al. (2019) found that bevacizumab combined with standard chemotherapy drugs led to a significant improvement in the progression-free survival (PFS), mainly of highrisk patients (stages III-IV) affected by ovarian cancer. However, some points such as the dosage, schedule, combination with other drugs, as well as the safety of bevacizumab in other dosages still need to be clarified. Even so, the study by Marchetti et al. (2019) has expanded the knowledge about the role of antiangiogenesis therapy in ovarian cancer.

As aforementioned, the high mortality of ovarian cancer is usually attributed to its unknown etiology and late detection. However, another 'lethal' feature of this kind of tumor is the fact that most patients relapse after 2 years of detection even after optimal cytoreductive surgery and standard adjuvant chemotherapy (Colombo et al. 2003). This relapse is due to cancer 
stem cells which, after chemotherapy, find a favorable environment less hypoxic where they can proliferate (Tomao et al. 2014). The second line of chemotherapy could be preceded by a secondary cytoreductive surgery. This could comprise a different combination of drugs; however, no benefits have been demonstrated compared to single-drug treatment (Giornelli 2016). In this context, bevacizumab is now being considered for the combination with carboplatin/gemcitabine, because it has shown some benefits in the overall response rate and PFS, and also because some patients experience relief in ascitis. For these reasons, although the benefits of this drug account for only 4 months in PFS (Giornelli 2016), bevacizumab has been approved for relapsed patients who had not received it in the first line. Nevertheless, two preclinical studies (OCEANS and AURELIA) have suggested that prolonged administration of bevacizumab as maintenance therapy after platinumbased chemotherapy prolongs PFS is well tolerated. However, the OCEANS trial, performed in platinumsensitive recurrent ovarian cancer, found no differences between overall survival when bevacizumab was added to the therapy (Aghajanian et al. 2015). In contrast, the AURELIA study, for platinum-resistant recurrent ovarian cancer, reported a significant increase in overall survival with the use of bevacizumab (Bamias et al. 2017). At present, guidelines give clear recommendations as to when and how to use bevacizumab in ovarian cancer treatment. However, taking into account its cost and its associated side effects, it is important to determine the proper use of this inhibitor as well as to identify the group of patients who can benefit from this therapy. Regarding angiogenesis-related drugs, until now, bevacizumab is the only one approved for ovarian cancer treatment.

Regarding other drugs, the poly (ADP-ribose) polymerase (PARP) inhibitor olaparib is the first personalized treatment recently approved for patients with high-grade serous ovarian cancer with a germline or somatic BRCA1 or BRCA2 mutation. Moreover, this drug could be received much earlier as a maintenance drug in patients that have responded to first-line platinumbased chemotherapy, and $60 \%$ of patients receiving olaparib have had no progression of cancer after 3 years vs $27 \%$ receiving the placebo (Washington et al. 2019).

\section{Angiogenesis and ovarian cancer success or failure of the treatments under study}

To interpret results of clinical trials with antiangiogenic drugs and to evaluate possible compounds that affect tumor angiogenesis and disrupt tumor growth, it is important to know the particular characteristics of the tumor vasculature. Tumor vasculature is exacerbated and overstimulated due to the high production of angiogenic factors by the tumor cells. The excessive ambition of the tumor for oxygen, nutrients as glucose, and factors that favor its growth leads the tumor to the development of a highly branched but poor and ineffective vasculature. The master key factor of this aberrant vasculature, but so convenient for the tumor, is hypoxia, one of the main conditions of tumors. Beyond stimulating VEGF, hypoxia regulates, among others, glycolysis, immune evasion, genomic instability, and limitless replication potential, all aspects that will help the phenotype of the tumor to be metastasic, invasive, and prone to acquire resistance to drugs and radiation (Hockel \& Vaupel 2001, Al Tameemi et al. 2019). Interestingly, in the tumor mass, hypoxia is heterogeneous, defining a normoxic, a hypoxic, and a necrotic region. The hypoxic region is where these adapted cells with all the malignant characteristics aforementioned are found. Also, in this region, the vasculature is dilated, scarcely covered with (or lacking) perivascular cells, tortuous, with exacerbated growth but nonfunctional, with poor endothelium and resulting in poor blood flow. The cells of this impoverished vascularization impair the delivery of therapeutic agents, thus favoring tumor development (Hockel \& Vaupel 2001).

Tumor angiogenesis is different from vasculogenesis as it develops from preexisting vascular beds. In this sense, vascular disrupting agents (VDAs) that target existing blood vessels are currently being developed and their combination with antiangiogenic compounds are being considered. An example of a VDA currently under study is fosbretabulin. This drug is being tested in combination with others like bevacizumab or pazopanib (a tyrosine kinase receptor inhibitor). According to the phase II GOG186i study, the combination of fosbretabulin and bevacizumab decreases disease progression in 2.5 months compared to bevacizumab alone. However, this combination also increases the rate of cardiac events by $18 \%$. This study was performed in platinumsensitive and platinum-resistant patients and, although the differences found in survival are, again, no longer than 4 months, the study concluded that this scheme of treatment should be encouraged (Monk et al. 2016b). This shows the strong necessity for new therapeutic strategies in ovarian cancer.

Another strategy currently under study is the one carried out by the TRINOVA-1 study, which has been the first in showing that an antiangiogenic therapy significantly delays the time to second progression (i.e. PFS-2) in women with recurrent ovarian cancer. This study compared Paclitaxel plus trebananib, a peptibody that blocks binding of ANGIOPOIETIN1 and 2 to TIE2, or plus placebo (Monk et al. 2016a). As known, angiopoietins and their Tie receptors are critical for vessel homeostasis and angiogenesis. Whereas ANG1 induces vessel maturation, ANG2 cooperates with other factors in the induction of the vessel sprouting and appears to antagonize ANG1 function by preventing its binding to TIE2 receptor (Potente et al. 2011). During tumor progression, angiopoietins exert crucial roles in the angiogenic switch, and increased expression of ANG2 
relative to ANG1 in tumors correlates with poor prognosis (Hata et al. 2004). The results of the TRINOVA-1 study showed that overall survival was not significantly longer in the intent-to-treat population, although it improved in patients with ascites receiving trebananib. PFS-2 confirming the PFS benefit associated with trebananib was maintained through the second disease progression (Monk et al. 2016a). Similarly, the TRINOVA-3 study assessed whether trebananib improved PFS when added to carboplatin and paclitaxel as first-line therapy in advanced epithelial ovarian, primary fallopian tube, or peritoneal cancer in phase III clinical trial, but showed no positive results or improvements for these ovarian tumor types (Vergote et al. 2019).

Another targeted molecule associated with angiogenesis and overexpressed in ovarian cancer is $\alpha 5 \beta 1$-integrin (Moffitt et al. 2019). The treatment of platinum-resistant patients or patients with advanced epithelial ovarian cancer with Volociximab, an antibody against this protein, was not significantly effective at preventing disease progression in a phase II clinical trial (NCT00516841) (Bell-McGuinn et al. 2011).

Currently, numerous studies are being carried out to find treatments for ovarian cancer. All these studies show the complexity of setting up a new therapeutic strategy due to the ability of the tumor to evade them and the unexpected outcome during tumorigenesis. Besides, in treatments, it is important to distinguish the 'significant different improvement from the statistical point of view vs the significant improvement from the biological point of view'.

Different studies have now shown that tumors can evade the therapeutic blockade of angiogenesis and several mechanisms have been described. As described in detail by van Beijnum et al. (2015), these mechanisms include: (1) redundancy in growth factors; (2) recruitment of bone marrow-derived cells; (3) participation of stromal cells as pericytes; (4) vessel cooption; (5) increased metastasis; and (6) mechanisms that contribute to the loss of activity of antiangiogenic drugs like glycosylation and endothelial cell heterogeneity (van Beijnum et al. 2015).

\section{A novel and different concept of angiogenesis targeting}

As aforementioned, when angiogenesis targeting was proposed as a method to treat solid tumors, it was believed that this strategy would overcome the acquisition of resistance of tumor cells to drugs. However, after four decades, several 'stones on the road' have been found. Some of these are those aforementioned and exposed in the review of Van Beijnum (2015) regarding mechanisms of antiangiogenesis resistance. However, in 2001, Jain proposed the novel mechanism of 'vascular normalization' relevant to all diseases that involved abnormal vascularity (Jain 2001). The novelty of this concept is that, rather than destroying tumor vasculature, it improves it, so as to help the drugs and oxygen to reach the tumor mass (Jain 2001). The concept is based on the knowledge that tumors develop several alternative pathways to overcome the shortage of oxygen supply. This mechanism of vascular normalization is driven by the hypoxia-inducible factor, particularly HIF1A, which is transcribed under hypoxic conditions to provide the cells with different strategies to survive in a hostile environment, as for example the stimulation of the transcription of genes that encode enzymes that participate in glycolysis (Al Tameemi et al. 2019).

In cancer therapy, when antiangiogenic therapy is applied, the process of vascular stabilization is accelerated. Regarding the mechanism of vascular normalization (Ehling \& Mazzone 2016) proposed the blockade of the excess of angiogenic stimuli to a level where the vasculature resembles a functional one. During tumor progression, the angiogenic vessels mature by the association of vascular mural cells which normalize the vascular structures. This is a dynamic and strictly regulated process. Because of the role of mural cells in vascular maturation, an adequate strategy to reach vessel normalization seems to be to target perivascular cells (pericytes and vascular smooth muscle cells). Several clinical studies have shown that the alteration of the vascular endothelium could also affect vascular beds in healthy tissues, resulting in negative side effects. In tumor vasculature, these perivascular cells are often loosely attached to endothelial cells and are reduced in number (Abramsson et al. 2003, Jain \& Booth 2003, Tong et al. 2004).

\section{Vascular normalization affects different parameters in tumor growth}

Tumor vessels are often poorly activated because of the constitutive proangiogenic signaling. This means that adhesion molecules and chemokines are downregulated, a fact that alters the recruitment of T-lymphocytes to the tumor tissue. When vascular normalization is in process, endothelial cells become activated and thus the number of cell adhesion molecules increases, providing an efficient barrier to liquids or cell extravasation. This mechanism arrests circulating immune cells prior to tissue extravasation and therefore improves leukocyte recruitment to the tumor tissue. Consequently, a possibility to enhance the efficiency of cancer immunotherapy could be to combine proinflammatory drugs with antiangiogenic therapy, which can enable tumor-targeted T-lymphocytes to access the tumor tissue (Klein 2018). In this sense, immunotherapy relies on the high activity of immune effector cells in the tumor microenvironment. Since May 2017, when the FDA approved an inhibitory antibody that targets the programmed cell death protein 1 (PD1) for certain solid tumors, the strategy of targeting immune components 
in tumors gained interest among clinical researchers. However, for unknown reasons, there is still a lack of responsiveness in the majority of patients that suffer from cancer. These unexplained results could be also due to tumor vasculature features (hypoxia, low $\mathrm{pH}$, and high interstitial fluid pressure), which, again, reduces the effectiveness of all cancer treatments, including immunotherapies. There are several evidences that suggest that a patient with ovarian cancer can benefit from immunotherapy, such as immune evasion mechanisms associated with poor survival and patients with T-cellrich tumors with longer PFS and overall survival (Ghisoni et al. 2019). Although different clinical trials are being conducted, no immunotherapeutic agent has obtained regulatory approval for epithelial ovarian cancer. These studies include combinations of the immune agent with anti-VEGF drugs or with PARP inhibitors. Regarding the latter, Lee et al. published a study that shows that PARP inhibitors and PD-1/PD-L1 blockade enhance the antitumor effect, compared to therapy with PARP inhibitors alone, without significantly increasing toxicity (Lampert et al. 2020). Hopefully, future upcoming results will clarify whether immunotherapy can be considered a new therapy for ovarian cancer.

Another characteristic of tumor vasculature is the high vessel compression, which strongly impairs blood flow and tumor oxygenation. Thus, vessel stabilization would lead to decompression of the vasculature and, consequently, a better oxygenation (among others) (Griffon-Etienne et al. 1999). In 1999, the first preclinical data showing vascular decompression and reduced hypoxia were those obtained with the use of the chemotherapeutic paclitaxel. Later, Alvarez et al. (2013) demonstrated that this effect was due to the ability of paclitaxel to reduce cancer-associated fibroblasts and collagen levels, an ability not present in other chemotherapeutic drugs. Zhao et al. (2019) have recently reported that the targeting of the angiotensin signaling axis with losartan, an approved angiotensin system inhibitor, enhances the efficacy of paclitaxel. This effect was achieved via normalizing the tumor microenvironment, which resulted in improved vessel perfusion and drug delivery as shown in two orthotopic human ovarian carcinoma xenograft models.

Schiffmann et al. (2017) also demonstrated that pharmacological inhibition of the VEGF pathway can reverse many of the unfavorable characteristics of the tumor vasculature, both in preclinical models and in cancer patients, and this explains the fact that VEGF inhibitors have been most successfully used in combination with chemotherapy.

Another mechanism to improve the contact between perivascular cells and endothelial cells in tumor vasculature is to interfere with factors like plateletderived growth factor (PDGF), angiopoietin-1 (ANG1), transforming growth factor- $\beta$ (TGFB) or sphingosine1-phosphate (S1P), which play important roles in the pericyte-endothelial cell interaction in physiological angiogenesis. In this sense, the inhibition of PDGF or its receptor has gained interest, and also in combination with VEGF inhibition. However, although numerous studies have evaluated the inhibition of PDGF or its receptor, this strategy has shown disappointing results. In particular, our laboratory is interested in PDGFB acting as a proangiogenic factor that induces angiogenesis to overcome hypoxia. This factor, which is released by endothelial cells, acts on PDGFB receptor-positive cells (pericytes) and chemoattracts them to the endothelium for its stabilization (Carmeliet \& Jain 2011). This action has been widely demonstrated in different animal models (Bjarnegard et al. 2004, Fruttiger 2007, Quaegebeur et al. 2010). Regarding this issue, our group achieved interesting results in an orthotopic experimental model of ovarian cancer in which we investigated the role of a Notch inhibitor, in combination with PDGFB as a normalization factor for ovarian cancer vasculature (Pazos et al. 2018). The Notch system regulates vessel branching and is also involved in tumor metastasis. In tumor angiogenesis, the blockade of this system results in an increased, but nonfunctional, vascularity with high vessel branching and sprouting. Interestingly, similar results have been obtained in anti-VEGF-resistant tumors (Noguera-Troise et al. 2006).

In our aforementioned work, we also observed that the administration of a gamma-secretase inhibitor (DAPT), which impairs Notch system activation, decreases tumor growth on day 6 in an ovarian cancer xenograft model and causes no changes in tumor vasculature stabilization markers such as $\alpha$-smooth muscle actin or PDGFB (perivascular markers) (Pazos et al. 2018). However, and according to the known role of the Notch system in angiogenesis, DAPT increased vascular permeability in a perfusion assay performed with fluorescent lectin in mouse ovarian tumors (Pazos et al. 2018). This inhibitor has an antitumor effect as its administration decreases proliferation markers like Ki67 and PCNA, as well as the intracellular pathway PI3K/AKT (Fig. 1) (Pazos et al. 2018). After the corroboration that, effectively, PDGFB administration to our ovarian cancer model increases perivascular coverage, decreases vascular permeability, and improves blood perfusion in tumor vasculature, we used this factor as the normalization agent in our orthotopic ovarian tumor model. Interestingly, the co-administration of DAPT and PDGFB prevented the tumor growth rate, which remained close to the initial size of control tumors (Fig. 1). The co-treatment improved all vascular parameters and turned DAPT treatment into a more efficient one (Fig. 1). This was also noted in molecular parameters related to proliferation and apoptosis (Pazos et al. 2018). Another evidence of the normalized vasculature in our study was the effect of the co-treatment on the VEGF and angiopoietins. The administration of DAPT and PDGFB decreased tumor VEGF levels, responsible for the 'angiogenic switch', 


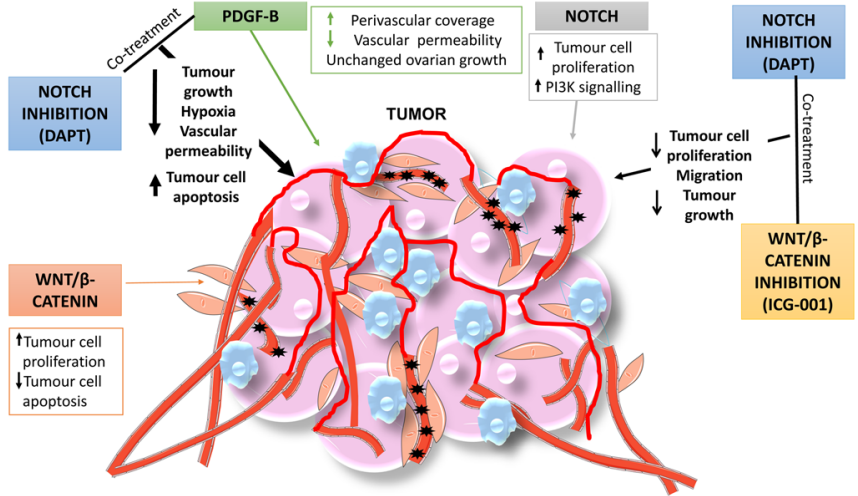

where new structurally and functionally abnormal vasculature is formed in and around the tumor, and in addition, increased tumor ANG1 and decreased tumor ANG2 (Pazos et al. 2018). It is well known that ANG1 stimulates mural coverage and basement membrane deposition, thereby promoting vessel tightness (Augustin et al. 2009). All this experimental evidence has shown that PDGFB could be considered as a normalizing agent in ovarian tumor vasculature.

PDGF-C, another member of the PDGF family, has been demonstrated to have maturation effects on the vasculature. di Tomaso et al. (2009) demonstrated that glioblastoma tumors which overexpressed PDGFC had smaller vessel diameters and lower vascular permeability and these tumors also had more perivascular coverage and a thicker basement membrane (di Tomaso et al. 2009). Further evidence that PDGFs may act to normalize vasculature has been demonstrated in breast cancer. Although in these tumors it was demonstrated that PDGFD overexpression promotes tumor growth, proliferation and metastasis, the overexpression of PDGFD increases perivascular coverage and normalizes tumor blood vessels, increasing doxorubicin penetration and thus, the treatment efficacy (Liu et al. 2011). However, before proposing the PDGF family members as normalizing factors, it is important to determine their precise effect on tumor cells.

\section{Alternative therapies for ovarian cancer to be explored}

Regarding alternative therapies for ovarian cancer, the inhibition of the $\mathrm{Wnt} / \beta$-catenin canonical pathway should be considered. The Wnt pathway cooperates with the epithelial-to-mesenchymal transition process induced by different compounds such as TGFB in ovarian cancer (Mitra \& Roy 2017, Niu et al. 2017).
Figure 1 Schematic representation of Notch and $\mathrm{Wnt} / \beta$-catenin effects on ovarian tumor biology. Both pathways promote ovarian tumor cell proliferation and migration as the individual inhibition of the signaling pathways decreases both parameters. However, when Notch and Wnt/ $\beta$-catenin are simultaneously inhibited, the effect achieved is similar to the independent inhibition, which shows that both pathways are not synergistic in ovarian cancer cell biology $(\rightarrow)$ (Bocchicchio et al. 2019). In ovarian cancer, PDGF acts as a normalization factor for tumor vasculature as observed in an in vivo model of xenograft mice. PDGF administration increases perivascular coverage and decreases vascular permeability, while ovarian growth remains unchanged. The co-treatment of PDGF and the Notch inhibitor DAPT enhances these effects, and also decreases tumor hypoxia and increases tumor cell apoptosis $(\rightarrow)$ (Pazos et al. 2018).

In addition, it has been shown that the activation of the $\mathrm{Wnt} / \beta$-catenin pathway is a critical driver of chemotherapy resistance (Nagaraj et al. 2015). In patients with primary ovarian cancer, $\mathrm{Wnt} / \beta$-catenin inhibition leads to a decrease in cellular proliferation and an increase in cellular apoptosis of the tumor cells isolated from ascites. In our laboratory, we have used two $\beta$-catenin inhibitors, XAV939 and ICG-001, in ovarian cancer cell lines in culture, and found that, although both inhibitors have a different mode of action, they decreased ovarian cancer cell proliferation and the expression of Wnt-associated proteins like Cyclin D1 and cMyc (Bocchicchio et al. 2019). In addition, the inhibitor ICG-001 markedly increased tumor cell apoptosis and inhibited ovarian tumor cell migration. The observed effects were achieved through a reduction in $\beta$-catenin protein levels and $\mathrm{Wnt} / \beta$-catenin pathway activation, as evidenced by the $\mathrm{Wnt} / \beta$-catenin target genes studied (Fig. 1) (Bocchicchio et al. 2019). Besides, the treatment with ICG-001 $(5 \mathrm{mg} / \mathrm{kg})$ in mice bearing ovarian tumor xenografts significantly decreased the tumor growth compared to untreated animals (Fig. 1). This effect was also evident in the proliferation of tumor cells, as demonstrated by a decrease in proliferating marker (Ki67)-positive cells (Bocchicchio et al. 2019). Interestingly, a clinical trial is currently being carried out to evaluate the safety and pharmacokinetics of orally administered SM08502 (NCT03355066). This molecule was shown to reduce Wnt pathway signaling and gene expression through potent inhibition of CDC-like kinase activity and is a novel strategy to reduce Wnt pathway signaling in tumors (Tam et al. 2020). These authors also found that SM08502 disrupted spliceosome activity, which was associated with inhibition of Wnt pathwayrelated gene and protein expression.

Regarding the $\mathrm{Wnt} / \beta$-catenin pathway, some other $W n t / \beta$-catenin inhibitors are under study for different 
types of tumors (Moore et al. 2019). In particular, a phase Ib clinical study has recently evaluated the doselimiting toxicities and the maximum tolerated dose of Ipafricept (also known as OMP-54F28) in ovarian cancer, where its efficacy was tested in combination with carboplatin and paclitaxel (Le et al. 2015, Harb et al. 2019). It has been described that the Wnt pathway is relevant in angiogenesis and vessel remodeling. This pathway regulates VEGF, interleukin-8, and matrix metalloproteinases (MMPs), which are key drivers and regulators of angiogenesis (Parmalee \& Kitajewski 2008, Zerlin et al. 2008). Thus, the inhibition of the $\mathrm{Wnt} / \beta$ catenin pathway in cancer could lead to the impairment of angiogenesis or its modulation could cause vascular stabilization. Now it is known that Wnt signaling is involved in tumor angiogenesis in solid tumors (Sherwood 2015). In particular, the inhibition of the ligand Wnt 1 by the Wnt inhibitory factor 1 (WIF1) significantly reduces the growth of hepatocellular carcinoma tumors in mice and prolongs animal survival (Hu et al. 2009). The authors associated this antineoplasic effect with reduced microvessel density and decreased VEGF expression (Hu et al. 2009). A similar effect has been demonstrated in cervical cancer where WIF1 was found to inhibit multiple critical events in tumor progression and metastasis, such as cell proliferation, angiogenesis and invasion (Ramachandran et al. 2012). Although several studies have shown the effect of the inhibition of the Wnt pathway in tumor angiogenesis, which turns Wnt inhibitors into VDA compounds beyond the known antineoplastic effect (Liu et al. 2016, Yang et al. 2019), few studies have been performed in ovarian cancer, a field that deserves attention.

Most of the actions mentioned for Wnt signaling are shared with the Notch system. This is now known for its very well-described role in multiple key aspects of tumor development (Aster et al. 2017). The first evidence was reported by Ellisen et al. (1991), who showed an overexpression of the human Notch homolog (TAN-1) in mouse and human lymphoid tissues and a translocation in the TAN-1 locus, which suggested the involvement of Notch in the transformation or progression of some T cell neoplasms (Ellisen et al. 1991). Nowadays, it is well demonstrated that Notch can be an oncogenic as well as a tumor suppressor pathway depending on the tumor type. In breast cancer, NOTCH1 receptor and JAGGED1 ligand have been unequivocally correlated with a poor overall survival in patients with advanced stages of this tumor (Reedijk et al. 2005). However, due to side effects of the Notch inhibitor, several studies are currently being carried out to determine the safety dose of these inhibitors in combinations with other chemotherapeutic drugs. This is the case of the phase Ib clinical trial of a gamma-secretase inhibitor in combination with gemcitabine in pancreatic ductal adenocarcinoma, in which it was determined that the combination of both drugs can be performed safely (Cook et al. 2018).
However, few studies have been performed in ovarian cancer. This pathway has been found changed in the high-grade serous histological subtype (Testa et al. 2018, Lisio et al. 2019). Also, upregulation of the Notch3 receptor gene and Jag2 ligand gene have been detected in ovarian cancer cell lines and also confirmed in another study by immunohistochemistry (Euer et al. 2005). Studies detecting other Notch members in human ovarian cancer specimens have also been conducted. Recently, by using bioinformatic tools, Jia et al. (2019) reported that several of the principal components of the Notch pathway, like the receptors $\mathrm{NOTCH} 2$ and $\mathrm{NOTCH} 3$, and the ligands DLL1, DLL3, DLL4, among others, are associated with poor overall survival in ovarian cancer. The numerous reports available regarding ovarian cancer and Notch signaling suggest the importance of evaluating this pathway as a therapeutic target. Regarding angiogenesis, it has been described that the ligands JAGGED1 and DLL4 play important roles in tumor and endothelial compartments of ovarian cancer mouse models (Mailhos et al. 2001, Steg et al. 2011). The effect of Notch in angiogenesis was very well described by Thurston et al., who demonstrated that too much angiogenesis caused by Notch inhibitors (non-functional angiogenesis) impairs tumor growth (Thurston et al. 2007). This report brought about new concepts about Notch inhibitors as antiangiogenic treatments. As a result of the huge amount of research done, some molecules have entered the clinical phase. In 2016, Pant et al. (2016) found a limited antitumor activity in solid tumors, including ovarian cancer; however, some of the patients had stable disease for the drug. These authors evaluated 35 patients with different types of tumors, so at the time of publication the study was very restrictive; however, more results will be shown when the clinical trial has ended. In a first human study of LY3039478 (a selective inhibitor of Notch activation) for metastasic cancer, Massard et al. (2018) reported clinical activity (tumor necrosis, metabolic response, and tumor shrinkage) in patients with breast cancer, leiomyosarcoma, and adenoid cystic carcinoma. However, more clinical research is needed to determine the efficacy of these inhibitors. Although these inhibitors are also being extensively studied for Alzheimer's disease treatment, some controversies have been found and other kinds of molecules are under development. Besides, clinical trials for some of them have been discontinued due to undesirable side effects (Kumar et al. 2018).

As aforementioned, the Notch and Wnt systems interact in different biological contexts as well as in the vascular system (Olsen et al. 2017). Besides the role of Notch in vascular branching and remodeling, it is known that Notch promotes vascular stabilization. It has been reported that one of the mechanisms involving Wnt signaling is the Notch induction of the expression of the Notch-regulated ankyrin repeated protein (NRARP) 
(Phng \& Gerhardt 2009). This protein limits Notch signaling and promotes $\mathrm{Wnt} / \beta$-signaling, which supports vascular stability, prevents endothelial cell retraction and improves intracellular cell junctions (Phng \& Gerhardt 2009). Many lines of evidence have shown the interaction between both pathways. In cancer, it has also been reported that there is a crosstalk between Wnt and Notch signaling. In skin cancer, Notch acts as a tumor suppressor by inhibiting Wnt signaling and the inhibition of Notch results in an increase in $\beta$-catenin (Chatterjee \& Sil 2019). Similarly, in neuroblastoma cells, Delta-Notch induces molecules that inhibit the Wnt signaling pathway (Dickkopf 1-3 (DKK1-3)) and secreted frizzled-related protein 1 (SFRP1) correlates with good prognosis of this tumor (Revet et al. 2010). The previously mentioned protein NRARP is also a link between both pathways in cancer. In T-cell acute lymphoblastic leukemia (T-ALL), where Notch is a known driver, NRARP is present in high levels. NRARP interacts with lymphoid enhancerbinding factor 1 (LEF1) and potentiates Wnt signaling in T-ALL cells with low levels of Notch, promoting the expansion of T-ALL cells (Pinto et al. 2020). LEF1 is a DNA-binding transcription factor that acts downstream of the Wnt signaling pathway by interacting with nuclear $\beta$-catenin (MacDonald et al. 2009). In ovarian cancer, no previous studies have evaluated the crosstalk between these two pathways, and this is thus a field to be investigated which can provide new alternative therapies. At our lab, we have evaluated the effect of the inhibition of the Notch and Wnt pathways, individually and simultaneously, in ovarian cancer cell lines, and have effectively detected an interaction between the Notch and Wnt pathways. In two different ovarian cell lines, the simultaneous inhibition of $\beta$-catenin and Notch signaling, by using the ICG-001 and DAPT inhibitors, decreased ovarian cancer cell proliferation to the same extent as targeting only the $\mathrm{Wnt} / \beta$-catenin pathway. A similar effect was observed in IGROV1 cell migration. In these ovarian cells, the inhibition of the $\mathrm{Wnt} / \beta$-catenin pathway increased the Notch target genes Hes 1 and Hey 1 and increased the expression of the Notch ligand Jagged1. These results show that Notch and $\beta$-catenin signaling cooperate in ovarian cancer to ensure the proliferation and migration of cells and that this could be achieved, at least partly, by the upregulation of the Notch Jagged1 ligand in the absence of Wnt signaling (Bocchicchio et al. 2019). In summary, these data show that the Wnt pathway crosstalks with Notch in ovarian cancer cell functions, which may have implications in ovarian cancer therapeutics.

\section{Conclusions}

Ovarian cancer is an intricate system in the biology of tumors in which the driver factors involved in growth and development are yet to be elucidated. It is clear now that several mechanisms, and more to be discovered, interplay in this cancer. Thus, the better knowledge we have of them, the more alternatives to develop new therapeutic strategies will be available. In this sense, it would be important to remember the words of Dr Bernardo Houssay, one of Argentine Nobel Prizes in Medicine (1947) 'Pure science is undoubtedly the source that ceaselessly feeds the applied techniques'. This means that a better understanding through basic research is needed to design drug strategies and to be able to introduce drugs into the clinic and combine them with existing therapies.

\section{Declaration of interest}

The author declares that there is no conflict of interest that could be perceived as prejudicing the impartiality of this review.

\section{Funding}

Agencia Nacional de Promoción Científica y Tecnológica (ANCyPT) PICT 2014-429 and 2016-315, the National Council for Scientific and Technical Research (CONICET PIP 177) and Roemmers Foundation, Argentina.

\section{Acknowledgements}

I thank Fundación Williams (Argentina) and Fundación René Barón (Argentina) for their support to the Instituto de Biología y Medicina Experimental (CONICET), Buenos Aires, Argentina. I especially thank my mentor, Dr Tesone, for her critical reading of this manuscript.

\section{References}

Abramsson A, Lindblom P \& Betsholtz C 2003 Endothelial and nonendothelial sources of PDGF-B regulate pericyte recruitment and influence vascular pattern formation in tumors. Journal of Clinical Investigation 112 1142-1151. (https://doi.org/10.1172/JCI18549)

Aghajanian C, Goff B, Nycum LR, Wang YV, Husain A \& Blank SV 2015 Final overall survival and safety analysis of OCEANS, a phase 3 trial of chemotherapy with or without bevacizumab in patients with platinumsensitive recurrent ovarian cancer. Gynecologic Oncology 139 10-16. (https://doi.org/10.1016/j.ygyno.2015.08.004)

Al Tameemi W, Dale TP, Al-Jumaily RMK \& Forsyth NR 2019 Hypoxiamodified cancer cell metabolism. Frontiers in Cell and Developmental Biology 7 4. (https://doi.org/10.3389/fcell.2019.00004)

Alvarez R, Musteanu M, Garcia-Garcia E, Lopez-Casas PP, Megias D, Guerra C, Muñoz M, Quijano Y, Cubillo A, Rodriguez-Pascual J et al. 2013 Stromal disrupting effects of nab-paclitaxel in pancreatic cancer. British Journal of Cancer 109 926-933. (https://doi.org/10.1038/ bjc.2013.415)

Aster JC, Pear WS \& Blacklow SC 2017 The varied roles of notch in cancer. Annual Review of Pathology 12 245-275. (https://doi.org/10.1146/ annurev-pathol-052016-100127)

Augustin HG, Koh GY, Thurston G \& Alitalo K 2009 Control of vascular morphogenesis and homeostasis through the angiopoietin-Tie system. Nature Reviews: Molecular Cell Biology 10 165-177. (https://doi. org/10.1038/nrm2639)

Bamias A, Gibbs E, Khoon Lee C, Davies L, Dimopoulos M, Zagouri F, Veillard AS, Kosse J, Santaballa A, Mirza MR et al. 2017 Bevacizumab with or after chemotherapy for platinum-resistant recurrent ovarian 
cancer: exploratory analyses of the Aurelia trial. Annals of Oncology 28 1842-1848. (https://doi.org/10.1093/annonc/mdx228)

Bell-McGuinn KM, Matthews CM, Ho SN, Barve M, Gilbert L, Penson RT, Lengyel E, Palaparthy R, Gilder K, Vassos A et al. 2011 A phase II, single-arm study of the anti-alpha5beta1 integrin antibody volociximab as monotherapy in patients with platinum-resistant advanced epithelial ovarian or primary peritoneal cancer. Gynecologic Oncology 121 273-279. (https://doi.org/10.1016/j.ygyno.2010.12.362)

Bjarnegard $M$, Enge $M$, Norlin J, Gustafsdottir S, Fredriksson S, Abramsson A, Takemoto M, Gustafsson E, Fassler R \& Betsholtz C 2004 Endothelium-specific ablation of PDGFB leads to pericyte loss and glomerular, cardiac and placental abnormalities. Development 131 1847-1857. (https://doi.org/10.1242/dev.01080)

Bocchicchio S, Tesone M \& Irusta G 2019 Convergence of Wnt and Notch signaling controls ovarian cancer cell survival. Journal of Cellular Physiology 234 22130-22143. (https://doi.org/10.1002/jcp.28775)

Carmeliet P \& Jain RK 2011 Molecular mechanisms and clinical applications of angiogenesis. Nature 473 298-307. (https://doi. org/10.1038/nature10144)

Chatterjee S \& Sil PC 2019 Targeting the crosstalks of Wnt pathway with Hedgehog and Notch for cancer therapy. Pharmacological Research 142 251-261. (https://doi.org/10.1016/j.phrs.2019.02.027)

Colombo N, Guthrie D, Chiari S, Parmar M, Qian W, Swart AM, Torri V, Williams C, Lissoni A, Bonazzi C et al. 2003 International Collaborative Ovarian Neoplasm Trial 1: a randomized trial of adjuvant chemotherapy in women with early-stage ovarian cancer. Journal of the National Cancer Institute 95 125-132. (https://doi.org/10.1093/jnci/95.2.125)

Cook N, Basu B, Smith DM, Gopinathan A, Evans J, Steward WP, Palmer D, Propper D, Venugopal B, Hategan M et al. 2018 A phase I trial of the gamma-secretase inhibitor MK-0752 in combination with gemcitabine in patients with pancreatic ductal adenocarcinoma. British Journal of Cancer 118 793-801. (https://doi.org/10.1038/bjc.2017.495)

di Tomaso E, London N, Fuja D, Logie J, Tyrrell JA, Kamoun W, Munn LL \& Jain RK 2009 PDGF-C induces maturation of blood vessels in a model of glioblastoma and attenuates the response to anti-VEGF treatment. PLoS ONE 4 e5123. (https://doi.org/10.1371/journal.pone.0005123)

Ehling M \& Mazzone M 2016 Vessel normalization in the spot-light of cancer treatment. Trends in Molecular Medicine 22 85-87. (https://doi. org/10.1016/j.molmed.2015.12.009)

Ellisen LW, Bird J, West DC, Soreng AL, Reynolds TC, Smith SD \& Sklar J 1991 TAN-1, the human homolog of the Drosophila notch gene, is broken by chromosomal translocations in T lymphoblastic neoplasms. Cell 66 649-661. (https://doi.org/10.1016/0092-8674(91)90111-b)

Euer NI, Kaul S, Deissler H, Mobus VJ, Zeillinger R \& Weidle UH 2005 Identification of L1CAM, Jagged2 and neuromedin $U$ as ovarian cancerassociated antigens. Oncology Reports 13 375-387. (https://doi. org/10.3892/or.13.3.375)

Folkman J 1971 Tumor angiogenesis: therapeutic implications. New England Journal of Medicine 285 1182-1186. (https://doi.org/10.1056/ NEJM197111182852108)

Folkman J, Long DM, Jr \& Becker FF 1963 Growth and metastasis of tumor in organ culture. Cancer 16 453-467. (doi:10.1002/10970142(196304)16:4<453::aid-cncr2820160407>3.0.co;2-y)

Fruttiger M 2007 Development of the retinal vasculature. Angiogenesis 10 77-88. (https://doi.org/10.1007/s10456-007-9065-1)

Ghisoni E, Imbimbo M, Zimmermann S \& Valabrega G 2019 Ovarian cancer immunotherapy: turning up the heat. International Journal of Molecular Sciences 20 2927. (https://doi.org/10.3390/ijms20122927)

Giornelli GH 2016 Management of relapsed ovarian cancer: a review. Springerplus 5 1197. (https://doi.org/10.1186/s40064-016-2660-0)

Goodman L 2004 Persistence-luck-avastin. Journal of Clinical Investigation 113 934. (https://doi.org/10.1172/JCl21507)

Griffon-Etienne G, Boucher Y, Brekken C, Suit HD \& Jain RK 1999 Taxaneinduced apoptosis decompresses blood vessels and lowers interstitial fluid pressure in solid tumors: clinical implications. Cancer Research $593776-3782$.

Harb J, Lin PJ \& Hao J 2019 Recent development of Wnt signaling pathway inhibitors for cancer therapeutics. Current Oncology Reports 2112. (https://doi.org/10.1007/s11912-019-0763-9)

Hata K, Nakayama K, Fujiwaki R, Katabuchi H, Okamura H \& Miyazaki K 2004 Expression of the angopoietin-1, angopoietin-2, Tie2, and vascular endothelial growth factor gene in epithelial ovarian cancer. Gynecologic Oncology 93 215-222. (https://doi.org/10.1016/j. ygyno.2003.12.031)

Hockel M \& Vaupel P 2001 Biological consequences of tumor hypoxia. Seminars in Oncology 28 (Supplement 8) 36-41.

Hu J, Dong A, Fernandez-Ruiz V, Shan J, Kawa M, Martinez-Anso E, Prieto J \& Qian C 2009 Blockade of Wnt signaling inhibits angiogenesis and tumor growth in hepatocellular carcinoma. Cancer Research 69 6951-6959. (https://doi.org/10.1158/0008-5472.CAN-09-0541)

Jain RK 2001 Normalizing tumor vasculature with anti-angiogenic therapy: a new paradigm for combination therapy. Nature Medicine 7 987-989. (https://doi.org/10.1038/nm0901-987)

Jain RK \& Booth MF 2003 What brings pericytes to tumor vessels? Journal of Clinical Investigation 112 1134-1136. (https://doi.org/10.1172/ $\mathrm{JCl} 20087$ )

Jia D, Underwood J, Xu Q \& Xie Q 2019 NOTCH2/NOTCH3/DLL3/MAML1/ ADAM17 signaling network is associated with ovarian cancer. Oncology Letters 17 4914-4920. (https://doi.org/10.3892/ol.2019.10170)

Klein D 2018 The tumor vascular endothelium as decision maker in cancer therapy. Frontiers in Oncology 8 367. (https://doi.org/10.3389/ fonc.2018.00367)

Kumar D, Ganeshpurkar A, Kumar D, Modi G, Gupta SK \& Singh SK 2018 Secretase inhibitors for the treatment of Alzheimer's disease: Long road ahead. European Journal of Medicinal Chemistry 148 436-452. (https:// doi.org/10.1016/j.ejmech.2018.02.035)

Lampert EJ, Zimmer A, Padget M, Cimino-Mathews A, Nair JR, Liu Y, Swisher EM, Hodge JW, Nixon AB, Nichols E et al. 2020 Combination of PARP inhibitor olaparib, and PD-L1 inhibitor durvalumab, in recurrent ovarian cancer: a proof-of-concept Phase II study. Clinical Cancer Research 26 4268-4279. (https://doi.org/10.1158/1078-0432.CCR-200056)

Le PN, McDermott JD \& Jimeno A 2015 Targeting the Wnt pathway in human cancers: therapeutic targeting with a focus on OMP-54F28. Pharmacology and Therapeutics 146 1-11. (https://doi.org/10.1016/j. pharmthera.2014.08.005)

Leung DW, Cachianes G, Kuang WJ, Goeddel DV \& Ferrara N 1989 Vascular endothelial growth factor is a secreted angiogenic mitogen. Science 246 1306-1309. (https://doi.org/10.1126/science.2479986)

Lisio MA, Fu L, Goyeneche A, Gao ZH \& Telleria C 2019 High-grade serous ovarian cancer: basic sciences, clinical and therapeutic standpoints. International Journal of Molecular Sciences 20 952. (https:// doi.org/10.3390/ijms20040952)

Liu J, Liao S, Huang Y, Samuel R, Shi T, Naxerova K, Huang P, Kamoun W, Jain RK, Fukumura D et al. 2011 PDGF-D improves drug delivery and efficacy via vascular normalization, but promotes lymphatic metastasis by activating CXCR4 in breast cancer. Clinical Cancer Research $\mathbf{1 7}$ 3638-3648. (https://doi.org/10.1158/1078-0432.CCR-10-2456)

Liu L, Zhi Q, Shen M, Gong FR, Zhou BP, Lian L, Shen B, Chen K, Duan W, Wu MY et al. 2016 FH535, a $\beta$-catenin pathway inhibitor, represses pancreatic cancer xenograft growth and angiogenesis. Oncotarget 7 47145-47162. (https://doi.org/10.18632/oncotarget.9975)

MacDonald BT, Tamai K \& He X 2009 Wnt/beta-catenin signaling: components, mechanisms, and diseases. Developmental Cell 17 9-26. (https://doi.org/10.1016/j.devcel.2009.06.016)

Mailhos C, Modlich U, Lewis J, Harris A, Bicknell R \& Ish-Horowicz D 2001 Delta4, an endothelial specific notch ligand expressed at sites of physiological and tumor angiogenesis. Differentiation; Research in Biological Diversity 69 135-144. (https://doi.org/10.1046/j.14320436.2001.690207.x)

Marchetti C, Muzii L, Romito A \& Benedetti Panici P 2019 Firstline treatment of women with advanced ovarian cancer: focus on bevacizumab. OncoTargets and Therapy 12 1095-1103. (https://doi. org/10.2147/OTT.S155425)

Massard C, Azaro A, Soria JC, Lassen U, Le Tourneau C, Sarker D, Smith C, Ohnmacht U, Oakley G, Patel BKR et al. 2018 First-in-human study of LY3039478, an oral Notch signaling inhibitor in advanced or metastatic cancer. Annals of Oncology 29 1911-1917. (https://doi.org/10.1093/ annonc/mdy244)

Mitra T \& Roy SS 2017 Co-activation of TGFbeta and Wnt signalling pathways abrogates EMT in ovarian cancer cells. Cellular Physiology and Biochemistry 41 1336-1345. (https://doi.org/10.1159/000464436) 
Moffitt L, Karimnia N, Stephens A \& Bilandzic M 2019 Therapeutic targeting of collective invasion in ovarian cancer. International Journal of Molecular Sciences 20 1466. (https://doi.org/10.3390/ijms20061466)

Monk BJ, Poveda A, Vergote I, Raspagliesi F, Fujiwara K, Bae DS, Oaknin A, Ray-Coquard I, Provencher DM, Karlan BY et al. 2016a Final results of a phase 3 study of trebananib plus weekly paclitaxel in recurrent ovarian cancer (TRINOVA-1): long-term survival, impact of ascites, and progression-free survival-2. Gynecologic Oncology 143 27-34. (https:// doi.org/10.1016/j.ygyno.2016.07.112)

Monk BJ, Sill MW, Walker JL, Darus CJ, Sutton G, Tewari KS, Martin LP, Schilder JM, Coleman RL, Balkissoon J et al. 2016b Randomized Phase II evaluation of bevacizumab Versus bevacizumab plus fosbretabulin in recurrent ovarian, tubal, or peritoneal carcinoma: an NRG Oncology/ Gynecologic Oncology Group study. Journal of Clinical Oncology 34 2279-2286. (https://doi.org/10.1200/JCO.2015.65.8153)

Moore KN, Gunderson CC, Sabbatini P, McMeekin DS, MantiaSmaldone G, Burger RA, Morgan MA, Kapoun AM, Brachmann RK, Stagg R et al. 2019 A phase $1 \mathrm{~b}$ dose escalation study of ipafricept (OMP54F28) in combination with paclitaxel and carboplatin in patients with recurrent platinum-sensitive ovarian cancer. Gynecologic Oncology 154 294-301. (https://doi.org/10.1016/j.ygyno.2019.04.001)

Nagaraj AB, Joseph P, Kovalenko O, Singh S, Armstrong A, Redline R, Resnick K, Zanotti K, Waggoner S \& DiFeo A 2015 Critical role of Wnt/ beta-catenin signaling in driving epithelial ovarian cancer platinum resistance. Oncotarget 6 23720-23734. (https://doi.org/10.18632/ oncotarget.4690)

Niu H, Zhou W, Xu Y, Yin Z, Shen W, Ye Z, Liu Y, Chen Y, Yang S, Xiang R et al. 2017 Silencing PPA1 inhibits human epithelial ovarian cancer metastasis by suppressing the $\mathrm{Wnt} /$ beta-catenin signaling pathway. Oncotarget $\mathbf{8}$ 76266-76278. (https://doi.org/10.18632/oncotarget.19346)

Noguera-Troise I, Daly C, Papadopoulos NJ, Coetzee S, Boland P, Gale NW, Lin HC, Yancopoulos GD \& Thurston G 2006 Blockade of Dll4 inhibits tumour growth by promoting non-productive angiogenesis. Nature 444 1032-1037. (https://doi.org/10.1038/ nature05355)

Olsen JJ, Pohl SÖ, Deshmukh A, Visweswaran M, Ward NC, Arfuso F, Agostino M \& Dharmarajan A 2017 The role of Wnt signalling in angiogenesis. Clinical Biochemist: Reviews 38 131-142.

Pant S, Jones SF, Kurkjian CD, Infante JR, Moore KN, Burris HA, McMeekin DS, Benhadji KA, Patel BKR, Frenzel MJ et al. 2016 A firstin-human phase I study of the oral Notch inhibitor, LY900009, in patients with advanced cancer. European Journal of Cancer 56 1-9. (https://doi. org/10.1016/j.ejca.2015.11.021)

Parmalee NL \& Kitajewski J 2008Wnt signaling in angiogenesis. Current Drug Targets 9 558-564. (https://doi.org/10.2174/138945008784911822)

Pazos MC, Sequeira G, Bocchicchio S, May M, Abramovich D, Parborell F, Tesone M \& Irusta G 2018 PDGFB as a vascular normalization agent in an ovarian cancer model treated with a gamma-secretase inhibitor. Journal of Cellular Physiology 233 5949-5961. (https://doi.org/10.1002/ jcp.26404)

Phng LK \& Gerhardt H 2009 Angiogenesis: a team effort coordinated by notch. Developmental Cell 16 196-208. (https://doi.org/10.1016/j. devcel.2009.01.015)

Pignata S, Scambia G, Ferrandina G, Savarese A, Sorio R, Breda E, Gebbia V, Musso P, Frigerio L, Del Medico P et al. 2011 Carboplatin plus paclitaxel versus carboplatin plus pegylated liposomal doxorubicin as first-line treatment for patients with ovarian cancer: the MITO-2 randomized phase III trial. Journal of Clinical Oncology 29 3628-3635. (https://doi.org/10.1200/JCO.2010.33.8566)

Pinto I, Duque M, Goncalves J, Akkapeddi P, Oliveira ML, Cabrita R, Yunes JA, Durum SK, Barata JT \& Fragoso R 2020 NRARP displays either pro- or anti-tumoral roles in T-cell acute lymphoblastic leukemia depending on Notch and Wnt signaling. Oncogene 39 975-986. (https:// doi.org/10.1038/s41388-019-1042-9)

Potente M, Gerhardt H \& Carmeliet P 2011 Basic and therapeutic aspects of angiogenesis. Cell 146 873-887. (https://doi.org/10.1016/j. cell.2011.08.039)

Przybycin CG, Kurman RJ, Ronnett BM, Shih IeM \& Vang R 2010 Are all pelvic (nonuterine) serous carcinomas of tubal origin? American Journal of Surgical Pathology 34 1407-1416. (https://doi.org/10.1097/ PAS.0b013e3181ef7b16)
Quaegebeur A, Segura I \& Carmeliet P 2010 Pericytes: blood-brain barrier safeguards against neurodegeneration? Neuron 68 321-323. (https://doi. org/10.1016/j.neuron.2010.10.024)

Ramachandran I, Thavathiru E, Ramalingam S, Natarajan G, Mills WK, Benbrook DM, Zuna R, Lightfoot S, Reis A, Anant S et al. 2012 Wnt inhibitory factor 1 induces apoptosis and inhibits cervical cancer growth, invasion and angiogenesis in vivo. Oncogene 31 2725-2737. (https://doi.org/10.1038/onc.2011.455)

Reedijk M, Odorcic S, Chang L, Zhang H, Miller N, McCready DR, Lockwood G \& Egan SE 2005 High-level coexpression of JAG1 and NOTCH1 is observed in human breast cancer and is associated with poor overall survival. Cancer Research 65 8530-8537. (https://doi. org/10.1158/0008-5472.CAN-05-1069)

Revet I, Huizenga G, Koster J, Volckmann R, van Sluis P, Versteeg R \& Geerts D 2010 MSX1 induces the Wnt pathway antagonist genes DKK1, DKK2, DKK3, and SFRP1 in neuroblastoma cells, but does not block Wnt3 and Wnt5A signalling to DVL3. Cancer Letters 289 195-207. (https://doi.org/10.1016/j.canlet.2009.08.019)

Santaballa A, Barretina P, Casado A, García Y, González-Martín A, Guerra E, Laínez N, Martinez J, Redondo A \& Romero I 2016 SEOM Clinical Guideline in ovarian cancer (2016). Clinical and Translational Oncology 18 1206-1212. (https://doi.org/10.1007/s12094-016-1588-8)

Schiffmann LM, Brunold M, Liwschitz M, Goede V, Loges S, Wroblewski M, Quaas A, Alakus H, Stippel D, Bruns CJ et al. 2017 A combination of low-dose bevacizumab and imatinib enhances vascular normalisation without inducing extracellular matrix deposition. British Journal of Cancer 116 600-608. (https://doi.org/10.1038/bjc.2017.13)

Sherwood V 2015 WNT signaling: an emerging mediator of cancer cell metabolism? Molecular and Cellular Biology 35 2-10. (https://doi. org/10.1128/MCB.00992-14)

Steg AD, Katre AA, Goodman B, Han HD, Nick AM, Stone RL, Coleman RL, Alvarez RD, Lopez-Berestein G, Sood AK et al. 2011 Targeting the notch ligand JAGGED1 in both tumor cells and stroma in ovarian cancer. Clinical Cancer Research 17 5674-5685. (https://doi.org/10.1158/10780432.CCR-11-0432)

Tam BY, Chiu K, Chung H, Bossard C, Nguyen JD, Creger E, Eastman BW, Mak CC, Ibanez M, Ghias A et al. 2020 The CLK inhibitor SM08502 induces anti-tumor activity and reduces Wnt pathway gene expression in gastrointestinal cancer models. Cancer Letters 473 186-197. (https:// doi.org/10.1016/j.canlet.2019.09.009)

Testa U, Petrucci E, Pasquini L, Castelli G \& Pelosi E 2018 Ovarian cancers: genetic abnormalities, tumor heterogeneity and progression, clonal evolution and cancer stem cells. Medicines 5 16. (https://doi. org/10.3390/medicines5010016)

Thurston G, Noguera-Troise I \& Yancopoulos GD 2007 The D elta paradox: DLL4 blockade leads to more tumour vessels but less tumour growth. Nature Reviews Cancer 7 327-331.

Tomao F, Papa A, Strudel M, Rossi L, Lo Russo G, Benedetti Panici P, Ciabatta FR \& Tomao S 2014 Investigating molecular profiles of ovarian cancer: an update on cancer stem cells. Journal of Cancer 5 301-310. (https://doi.org/10.7150/jca.8610)

Tong RT, Boucher Y, Kozin SV, Winkler F, Hicklin DJ \& Jain RK 2004 Vascular normalization by vascular endothelial growth factor receptor 2 blockade induces a pressure gradient across the vasculature and improves drug penetration in tumors. Cancer Research 64 3731-3736. (https://doi.org/10.1158/0008-5472.CAN-04-0074)

Torre LA, Trabert B, DeSantis CE, Miller KD, Samimi G, Runowicz CD, Gaudet MM, Jemal A \& Siegel RL 2018 Ovarian cancer statistics, 2018. CA: A Cancer Journal for Clinicians 68 284-296. (https://doi. org/10.3322/caac.21456)

van Beijnum JR, Nowak-Sliwinska P, Huijbers EJ, Thijssen VL \& Griffioen AW 2015 The great escape; the hallmarks of resistance to antiangiogenic therapy. Pharmacological Reviews 67 441-461. (https:// doi.org/10.1124/pr.114.010215)

Vergote I, Scambia G, O'Malley DM, Van Calster B, Park SY, Del Campo JM, Meier W, Bamias A, Colombo N, Wenham RM et al. 2019 Trebananib or placebo plus carboplatin and paclitaxel as first-line treatment for advanced ovarian cancer (TRINOVA-3/ ENGOT-ov2/GOG-3001): a randomised, double-blind, phase 3 trial. Lancet: Oncology 20 862-876. (https://doi.org/10.1016/S14702045(19)30178-0) 
Wang S, Qiu L, Lang JH, Shen K, Yang JX, Huang HF, Pan LY \& Wu M 2013 Clinical analysis of ovarian epithelial carcinoma with coexisting pelvic endometriosis. American Journal of Obstetrics and Gynecology 208 413.e1-413.e5. (https://doi.org/10.1016/j.ajog.2012.12.004)

Washington CR, Richardson DL \& Moore KN 2019 Olaparib in the treatment of ovarian cancer. Future Oncology 15 3435-3449. (https:// doi.org/10.2217/fon-2019-0271)

Yang P, Yu D, Zhou J, Zhuang S \& Jiang T 2019 TGM2 interference regulates the angiogenesis and apoptosis of colorectal cancer via Wnt/ $\beta$-catenin pathway. Cell Cycle 18 1122-1134. (https://doi.org/10.1080/15384101 .2019.1609831)

Zerlin M, Julius MA \& Kitajewski J 2008 Wnt/frizzled signaling in angiogenesis. Angiogenesis 11 63-69. (https://doi.org/10.1007/s10456008-9095-3)
Zhao Y, Cao J, Melamed A, Worley M, Gockley A, Jones D, Nia HT, Zhang Y, Stylianopoulos T, Kumar AS et al. 2019 Losartan treatment enhances chemotherapy efficacy and reduces ascites in ovarian cancer models by normalizing the tumor stroma. PNAS 116 2210-2219. (https://doi.org/10.1073/pnas.1818357116)

Received 6 December 2019

First decision 23 January 2020

Revised Manuscript received 30 September 2020

Accepted 9 October 2020 